\title{
La baignade en rivière : comment concilier les divers usages de l'eau en milieu naturel pour garantir la bonne qualité de l'eau de baignade ? Le cas du bassin de la Semois en Région wallonne de Belgique
}

\author{
Francis RosiLlon et Carine MARCHAL \\ Université de Liège - Département des Sciences et Gestion de l'Environnement, \\ 185 avenue de Longwy, 6700 Arlon, Belgique \\ E-mails : f.rosillon@ulg.ac.be ; cmarchal@ulg.ac.be
}

(Article reçu le 6 octobre 2009 ; accepté le 10 janvier 2010)

\begin{abstract}
Résumé - La pratique de la baignade dans les eaux naturelles européennes est permise et soumise à la Directive 2006/7/CE relative à la qualité des eaux de baignade. En Région wallonne de Belgique, 36 sites aménagés en rivières et en lacs permettent cette activité en pleine nature.

Étant donné que les rivières sont partagées par de multiples usagers, la complexité du milieu, les pressions diverses sur les ressources hydriques et la cohabitation avec d'autres usages de l'eau conduisent au risque de ne pouvoir garantir en permanence, durant la saison estivale, une qualité satisfaisante de toutes les eaux de baignade.

Le modèle wallon de contrat de rivière offre un espace de rencontre entre tous les usagers de la rivière en prenant en compte les préoccupations de chacun en vue d'une gestion intégrée des cours d'eau. Cette approche basée sur la recherche d'un consensus entre les acteurs est traduite dans un programme d'actions concrètes. Trois actions du contrat de rivière Semois en faveur de la baignade sont présentées. Les deux premières actions conduisent à une amélioration de la qualité bactériologique de l'eau, la troisième permet de garantir un niveau d'eau suffisant pour permettre la baignade.

La première action a donc permis d'améliorer la qualité bactériologique de la rivière. Les rivières étant les milieux récepteurs des eaux usées, une source importante de contamination provient du rejet des effluents même après une station d'épuration classique avec traitement biologique. Si le système de désinfection des eaux par UV est régulièrement appliqué en Région wallonne, l'installation pilote d'un taillis de saules à courte rotation en tant que support à un traitement tertiaire d'eaux usées domestiques a permis de constater un excellent abattement de la charge bactérienne.
\end{abstract}


À côté de résultats satisfaisants en termes de réduction des charges en nitrates et phosphates, le taillis de saules permet de répondre aux normes de qualité des eaux de baignade.

La seconde réalisation concerne l'abreuvement du bétail en cours d'eau qui constitue un facteur de risque non négligeable en milieu rural. Une nouvelle réglementation oblige la pose de clôtures le long des cours d'eau localisés en zone pâturée par le bétail dans un périmètre de $10 \mathrm{~km}$ en amont de la zone de baignade, mais il s'agit aussi de préserver l'usage " abreuvement du bétail ». Une étude comparative, entre un ruisseau protégé par des clôtures avec l'installation de bacs abreuvoirs pour l'alimentation en eau du bétail et un cours d'eau non protégé dans lequel le bétail divague, a permis de noter des abattements de l'ordre de 2 à 3 unités logarithmiques des germes de contamination fécale.

La troisième action est relative à la restauration d'un ancien barrage à valeur patrimoniale et paysagère en vue de maintenir un niveau d'eau suffisant pour permettre la baignade dans l'un des huit sites en rivière Semois officiellement reconnus.

Ces trois réalisations, qui ont été initiées dans le cadre d'un contrat de rivière, permettent de démontrer l'intérêt et la possibilité de concilier divers usages de l'eau et de répondre aux préoccupations des usagers, notamment des adeptes de la baignade en rivière.

Au final, par l'organisation annuelle des journées européennes de la baignade qui se déroulent chaque année en Région wallonne, cette pratique populaire peut constituer un excellent support de sensibilisation du grand public à la bonne qualité des eaux de surface, tout en apportant un soutien citoyen à la mise en œuvre de la directive cadre eau.

Mots-clés : Baignade, rivière, contrat, qualité bactériologique, Wallonie, Semois

\footnotetext{
Abstract - Riverbathing: How to reconcile the different water uses in a natural environment in order to guarantee the good quality of bathing water? Case study: The Semois basin in the Walloon Region of Belgium. The practice of the bathing in European natural waters is allowed and subjected to the Directive 2006/7/CE relative to the quality of bathing waters. In Walloon Region of Belgium, 36 sites fitted out in river and in lakes allow this activity.

But the rivers are shared by multiple users and the different pressures on the water resources lead at the risk of not being able to guarantee permanently, during the summer season, a satisfactory quality of all the bathing waters.

The Walloon model of river contract offers a space of meeting between all the users of the river by taking into account the concerns of each with the aim of a integrated management. This approach based on the research for a consensus between the actors is translated in a plan of concrete actions. Three actions of the river contract Semois in favour of the bathing are presented.

The first action allowed to improve the bacteriological quality of the river by the implementation of a pilot-plant of a willows coppice as support in a disinfection treatment of domestic waste water. The willows coppice allows to answer the standards of bathing waters. The second
} 
realization concerns the cattle watering in the water course which constitutes a not unimportant risk factor in rural areas. A comparative study between a river where the cattle watering is controlled and a river where the access of the cattle is free has allowed to note a reduction of the order of 2 in 3 logarithmic units of faecal contamination bacteria. The third action consists of the restoration of an ancient dam to allow the bathing in one of eight sites in river Semois by the increasing of the water level.

These three achievements which were introduced within the framework of a river contract allow to demonstrate the interest and the possibility of reconciling different water uses and of answering the preoccupations of the users, in particular the fans of the riverbathing.

Keywords : Bathing, river, contract, bacteriological quality, Wallonia, Semois

\section{INTRODUCTION}

La pratique de la baignade dans les eaux naturelles européennes est permise et soumise à la Directive 2006/7/CE (Journal officiel de l'union européenne, 2006) concernant la gestion de la qualité des eaux de baignade en remplacement de la Directive précédente 76/160/CEE. En Région wallonne de Belgique, l'arrêté du Gouvernement wallon du 14 mars 2008 modifiant le Code de l'eau et relatif à la qualité des eaux de baignade transpose la récente directive européenne. On compte en Wallonie 36 sites aménagés en rivière et en lacs qui permettent cette activité en pleine nature.

Étant donné que les rivières et plans d'eau sont partagés entre de multiples usagers, la complexité du milieu, les pressions diverses sur les ressources hydriques et la cohabitation avec d'autres fonctions et usages de l'eau conduisent au risque de ne pouvoir garantir en permanence, durant la saison estivale, une qualité satisfaisante de toutes les eaux de baignade. La prise en compte de toutes ces fonctions et usages de la rivière, dont la pratique de la baignade, peut avoir lieu au sein du contrat de rivière.

Le contrat de rivière, comme outil de gestion de l'eau, basé sur un mode concerté en partenariat avec les acteurs publics et privés d'un bassin versant, est apparu en Région wallonne au début des années 90 (Rosillon et Vander Borght, 2005b). À cette époque, face à une gestion sectorielle de l'eau qui avait montré ses limites en termes d'efficacité environnementale et de développement durable, de nouvelles exigences sont apparues. Celles-ci, émises par divers acteurs de l'eau mais également par les forces vives de la société civile, ont fait percevoir un besoin de rupture avec un mode de gestion classique ne pouvant apporter des réponses satisfaisantes. Le souhait d'une nouvelle approche concertée était notamment justifié par la multiplication des usages de l'eau, en particulier dans le domaine des loisirs, qui conduisait à augmenter les pressions sur les ressources en induisant des conflits d'usages.

Le contrat de rivière est défini comme étant un protocole d'accord entre les acteurs publics et privés d'un bassin versant en vue de concilier les multiples fonctions et usages des cours d'eau, de leurs abords et des ressources en eau du bassin. Il est traduit dans un programme d'actions de restauration de la qualité des eaux.

Tout en répondant au souhait de maintenir une qualité d'eau de baignade, trois des actions du contrat de rivière Semois ont permis de mettre en exergue le caractère 
plurifonctionnel de la rivière et l'intérêt de concilier les différents usages pouvant contrarier la qualité microbiologique des eaux.

\section{Contexte : la baignade en rivière en Wallonie}

N'ayant pas accès à la mer, la Wallonie, région d'Europe ayant le plus dense réseau de cours d'eau (12 000 cours d'eau sur un territoire de $16844 \mathrm{~km}^{2}$ - Service public de Wallonie, 2008), a considéré les rivières comme un des atouts touristiques en faveur de son développement économique. Depuis de nombreuses années, ces eaux intérieures sont utilisées pour la pratique de la baignade en pleine nature. Les 36 zones de baignade officiellement désignées par le Gouvernement wallon (Arrêtés du Gouvernement Wallon du 24/7/2003, 27/5/2004, 29/6/2006 et 14/3/2008) sont réparties en 18 tronçons de rivière et 18 lacs et étangs.

La qualité de ces zones de baignade est contrôlée chaque semaine en saison estivale $^{1}$. Au-delà du suivi relatif à la qualité des zones de baignade, le législateur a mis en place une série de mesures préventives afin de protéger ces zones de baignade. Ces mesures sont reprises dans le programme des mesures imposé par la Directive Cadre Eau (DCE) dans la rubrique zones protégées : les zones de baignade. Ce sont des mesures de base portant sur (Service Public de Wallonie, 2009) :

- la désignation des zones de protection à l'amont des zones de baignade où un inventaire de toutes les sources de rejets a été effectué ;

- la poursuite de l'analyse bimensuelle de la qualité bactériologique des zones de baignade ;

- la révision périodique des inventaires des sources d'apport dans les zones d'amont ;

- la réduction des sources d'apports ponctuels dans les zones de protection amont et les zones de baignade ;

- l'interdiction de l'accès du bétail à tous les cours d'eau en amont des zones de baignade.

Signalons que la zone de protection amont peut s'étendre sur une distance de $10 \mathrm{~km}$ le long du cours d'eau principal mais aussi le long de ses affluents. Dans cette zone amont, les mesures de base se traduisent notamment par l'épuration avec désinfection des eaux usées et la réduction de l'impact du bétail s'abreuvant dans les cours d'eau.

Trente-quatre zones de baignades et leur partie amont ont été désignées par l'Arrêté ministériel du 27/04/2007 comme étant des zones prioritaires en matière d'assainissement autonome ${ }^{2}$. En matière d'épuration collective, l'épuration des habitations des agglomérations concernées par cette zone amont est prioritaire. Les dispositifs d'épuration comportent un traitement de désinfection. Dans la plupart

\footnotetext{
${ }^{1}$ Selon le tableau de bord de l'environnement wallon en 2008 (Service public de Wallonie, 2008), la qualité des eaux de baignade s'est améliorée de 1990 à 2005. En 2006 et 2007, 14 zones de baignade étaient non conformes et en termes de proportion d'échantillons non-conformes toutes stations confondues, $12 \%$ des échantillons sont concernés. En 2008, 13 zones étaient déclarées non-conformes (Lutgen, 2009).

${ }^{2}$ Dans ces zones, il y a obligation d'épurer les eaux avec l'installation d'un traitement de désinfection pour les stations de plus de 20 équivalents habitant (EH). Les installations touristiques, nombreuses en bordure des cours d'eau wallons dans lesquels se pratique la baignade, sont particulièrement concernées par cette obligation.
} 
des cas, c'est un dispositif de désinfection par UV qui est installé. Concernant la prévention des contaminations dues au bétail, la pose de clôtures en prairies pâturées en bord de cours d'eau est obligatoire pour les prairies localisées dans les zones amont.

Parmi les 36 zones wallonnes de baignade, huit sont localisées dans le bassin de la Semois : sept en rivière et une au niveau d'un lac. La qualité est relativement bonne. En 2008, un seul site en rivière était déclassé en présentant au moins deux échantillons non conformes. Mais étant donné le caractère rural agricole et touristique du bassin de la Semois, les mesures préventives adaptées à ce contexte trouvent ici leur application. L'agriculture essentiellement orientée vers l'élevage de bovins joue un rôle non négligeable en matière de qualité des eaux de surface tandis que les 55 campings installés dans la vallée de la Semois sont soumis au traitement de leurs eaux usées avant leur rejet dans la rivière. À côté de ces aspects qualitatifs, certains sites en Semois ne sont plus accessibles en saison estivale et notamment en période d'étiage pour des raisons de niveau d'eau insuffisant. Il faudra donc également prendre en compte cette problématique pour permettre la baignade.

\section{MATÉRIEL ET MÉTHODES}

\section{Le modèle wallon de contrat de rivière}

À partir de l'exemple français de contrat de rivière né dix années plus tôt (Ministère de l'écologie et al., 2009), la Région wallonne s'est donc dotée d'une première circulaire ministérielle en mars 1993. Selon les termes de cette circulaire, le modèle wallon de contrat de rivière est défini comme étant « un protocole d'accord entre l'ensemble des acteurs publics et privés sur des objectifs visant à concilier les multiples fonctions et usages des cours d'eau, de leurs abords et des ressources en eau du bassin ». Cette approche a été renforcée par l'intégration du contrat de rivière dans le code wallon de l'eau décidée par le gouvernement wallon en application de la DCE. En novembre 2008, un arrêté du Gouvernement wallon apportait une nouvelle base réglementaire au contrat de rivière, tout en reconnaissant son rôle dans la mise en œuvre de la DCE (Gouvernement wallon, 2008).

Le comité de rivière est la structure porteuse du projet. Il est désormais constitué de trois collèges regroupant les représentants des communes, des administrations et des acteurs locaux (usagers et ONG). Les membres du comité définissent consensuellement un programme d'actions de restauration des fonctions et usages de l'eau en tenant compte des préoccupations de chacun des acteurs, dans le respect des potentialités locales. Ces actions seront ensuite mises en œuvre par chacun des membres du comité en fonction de leurs responsabilités. Alors que la recherche du consensus est une priorité du contrat de rivière, celui-ci produit au quotidien ses effets, tant sur le plan environnemental, économique que social. Dès l'instant où un certain nombre de conditions sont réunies, dont l'acceptation par les acteurs d'une démarche participative et consensuelle, l'outil contrat de rivière peut être utilisé quel que soit le lieu.

Il existe actuellement 22 contrats de rivière en Wallonie, couvrant plus de $85 \%$ du territoire. Parmi ceux-ci, nous avons choisi le contrat du bassin de la Semois pour illustrer concrètement la démarche (Rosillon et al., 2009). À partir d'une initiative wallonne soutenue par les 12 principales communes belges du bassin, un premier 


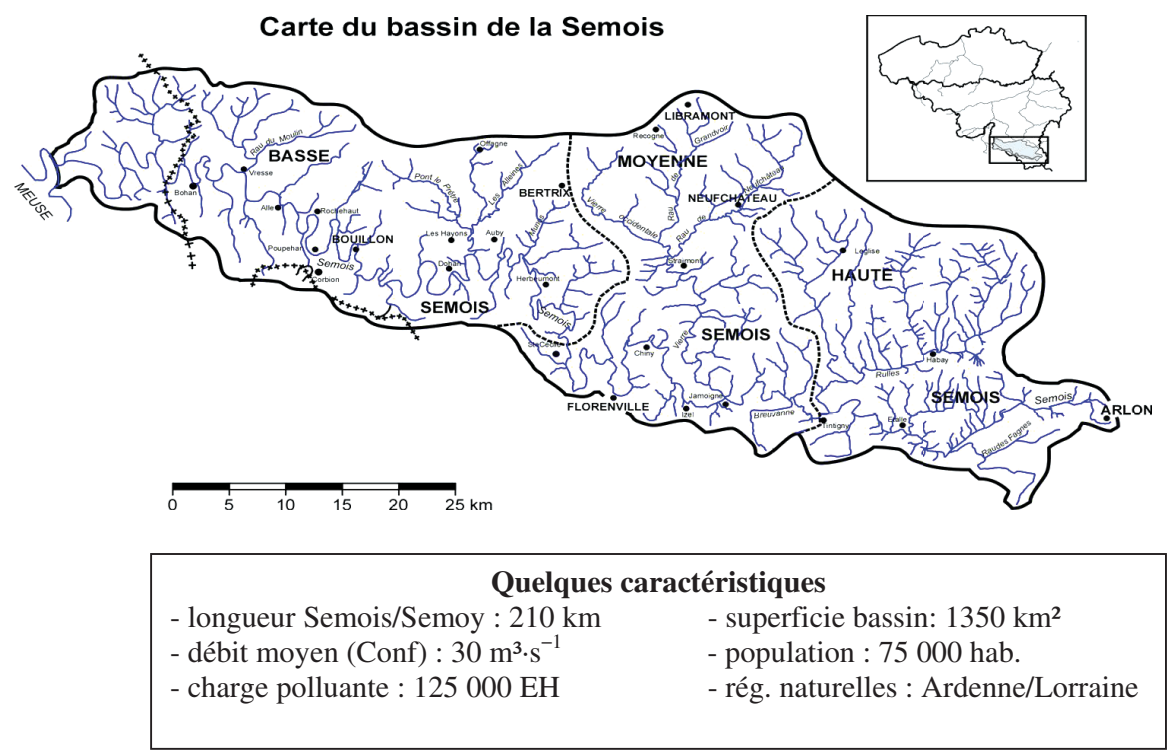

Fig. 1. Le bassin transfrontalier de la Semois (FUL - Contrat de rivière Semois, 1993).

Fig. 1. The Semois transboundary basin.

contrat signé en décembre 1996 a pu être élaboré. Celui-ci concernait la partie belge du bassin. À la faveur du programme Interreg II et III, Wallonie-ChampagneArdenne, la démarche a pu s'étendre en France (voir fig. 1).

Suite à l'application avec succès du premier programme d'actions (1996-2001), le comité de rivière a décidé d'élaborer un second contrat. Celui-ci est constitué de 220 actions réparties en 4 volets et 10 thèmes (voir fig. 2) :

- Volet I : Les connaissances

- Volet II : Les actions de restauration des fonctions et usages

- Volet III : La communication

- Volet IV : Le suivi et l'évaluation.

Le plus important, le volet II, porte sur une liste d'actions spécifiques et locales à mettre en œuvre sur le terrain. Les trois premiers thèmes sont essentiellement consacrés aux fonctions naturelles des cours d'eau et des milieux liés à l'eau tandis que les deux suivants s'intéressent plutôt aux usages et aux activités humaines. Le volet central II est encadré par trois autres domaines d'ordre général qui reflètent les autres missions d'encadrement du contrat de rivière : l'amélioration des connaissances, la communication, le suivi et l'évaluation.

Si la pratique de la baignade est affectée au thème 6 relatif aux activités économiques touristiques, celle-ci est aussi en lien avec les aspects qualitatifs et même, comme nous le verrons dans les exemples d'actions présentés ci-après, avec la fonction hydraulique et écologique et même avec des aspects liés aux zones protégées et à la protection du patrimoine. À travers la baignade, le contrat de rivière invite à une approche intégrée de la qualité des cours d'eau. 


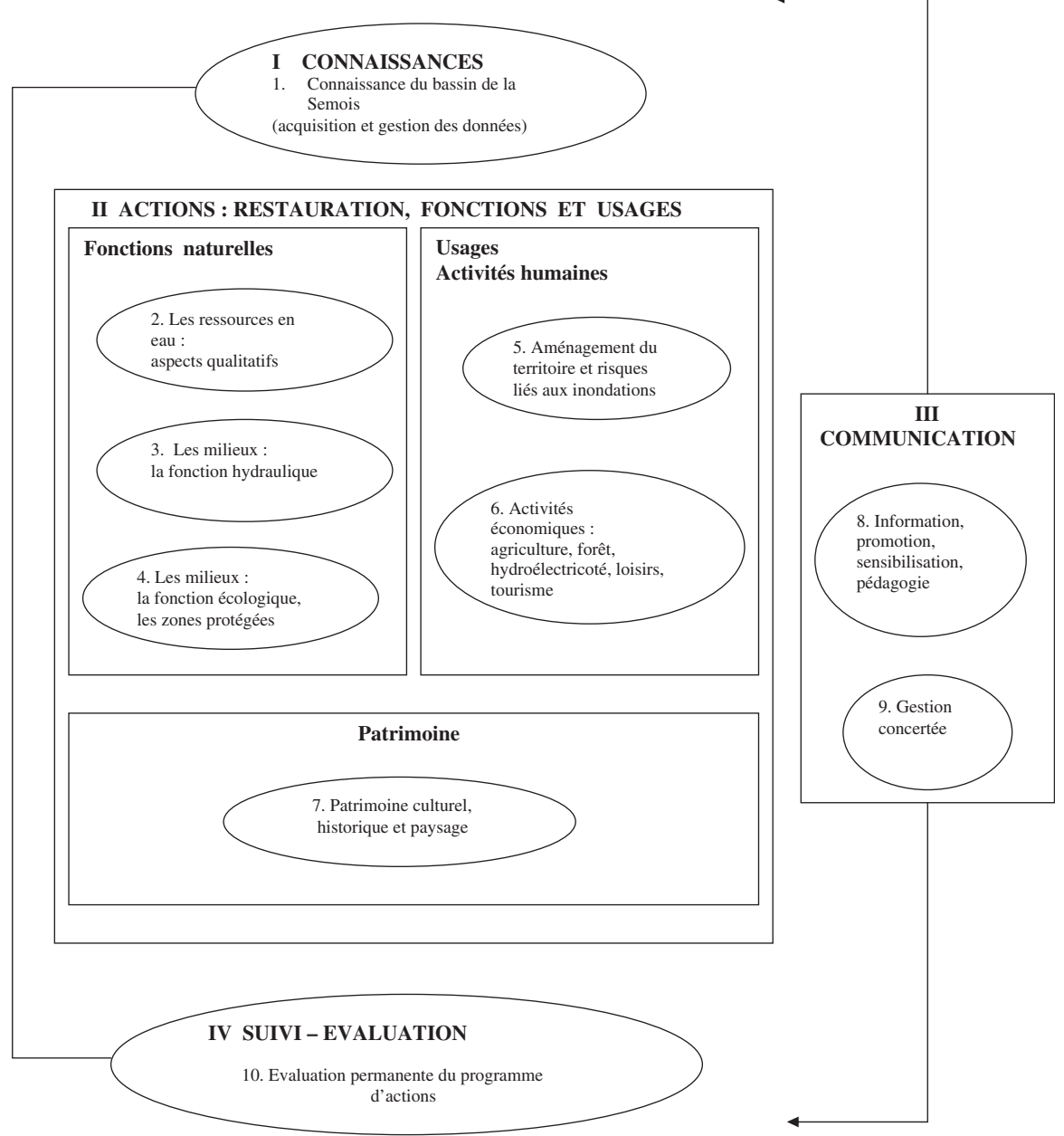

Canevas du programme d'actions : organisation des 10 thèmes

Fig. 2. Schéma d'organisation du contrat de rivière Semois.

Fig. 2. Organization of the Semois river contract.

\section{La qualité bactériologique des eaux dans le bassin de la Semois}

Dans l'esprit du contrat de rivière, l'évaluation de la qualité des eaux de surface ne peut être appréhendée qu'à travers une vision plurifonctionnelle de la rivière. Cette approche intégrée est confirmée par la DCE qui élargit la notion de qualité des eaux à une dimension écologique. Rappelons qu'un des objectifs de la DCE est justement l'atteinte d'un bon état chimique et écologique des eaux à l'horizon 2015, l'état écologique étant la résultante d'une qualité physico-chimique 
en support à la biologie, d'une qualité hydromorphologique du cours d'eau et d'une qualité biologique documentée par l'emploi des bio-indicateurs. Dans ce sens, le système d'évaluation de la qualité des eaux de rivière appelé SEQ-EAU (Simonet, 2001), constitue un outil adapté à ce type d'approche. À des classes d'altération de la qualité physico-chimique, correspondent des classes d'aptitudes de l'eau à la biologie et aux usages dont celui lié aux loisirs et à la baignade.

En complément du suivi officiel du service public de wallonie, le laboratoire du département en sciences et gestion de l'environnement de l'ULG effectue, pour le compte du contrat de rivière Semois, des campagnes d'analyses en lien avec les actions réalisées. C'est ainsi que des campagnes ont été menées à propos de l'impact du bétail en cours d'eau et du suivi de stations d'épuration, notamment à propos de la qualité bactériologique des eaux.

La méthode par incorporation en gélose et par filtration lorsque les eaux sont peu contaminées a été retenue pour l'analyse bactériologique. Les indicateurs de contamination fécale analysés ont été : Escherichia coli et coliformes fécaux par utilisation du milieu Rapid' Ecoli, les entérocoques fécaux par ensemencement du milieu $\mathrm{D}$-Coccosel bile esculine. Après incubation à $44,5^{\circ} \mathrm{C}$ pendant $24 \mathrm{~h}$, les colonies ont été comptées, les résultats étant exprimés en nombre d'UFC/100 mL d'eau.

\section{Présentation des trois dispositifs étudiés en lien avec l'usage baignade}

\section{- Un taillis de saules pour la désinfection des eaux usées}

Un dispositif d'épuration par taillis de saules a pu voir le jour grâce au contrat de rivière qui constitue un terrain favorable à l'émergence de techniques innovantes. Celui-ci a été installé en vue de compléter l'épuration d'un village de $500 \mathrm{EH}$ situé dans le bassin de la Semois (le village de Saint-Vincent, Commune de Tintigny) dans le but de mieux protéger le milieu récepteur des eaux après épuration. Ce milieu est en fait une réserve naturelle, les marais de Prouvy, un site intéressant au niveau de la biodiversité notamment par la présence de plantes rares inféodées à ce milieu humide à caractère oligotrophe (Rosillon et al., 2004).

Ces marais sont alimentés par plusieurs cours d'eau. L'un de ces cours d'eau reçoit les eaux épurées de la station d'épuration de type «boues activées » du village. Bien que respectant dans l'ensemble les normes de rejet, la station émettait des eaux eutrophes qui risquaient à terme d'entraîner une perte de biodiversité et une banalisation de la végétation au niveau des marais classés en zone Natura 2000.

Le taillis de saules, installé sur un sol en place, constitue un filtre végétal. Le système sol-plante peut être défini comme un réacteur bio-chimio-physique où les particules du sol, les microorganismes et la plante ont un rôle à jouer respectivement dans les processus d'échanges d'ions, dans la dégradation des matières organiques et dans la consommation d'éléments nutritifs. Deux mares réceptionnent les eaux sortant du taillis de saules avant leur rejet dans le ruisseau (voir fig. 3). 


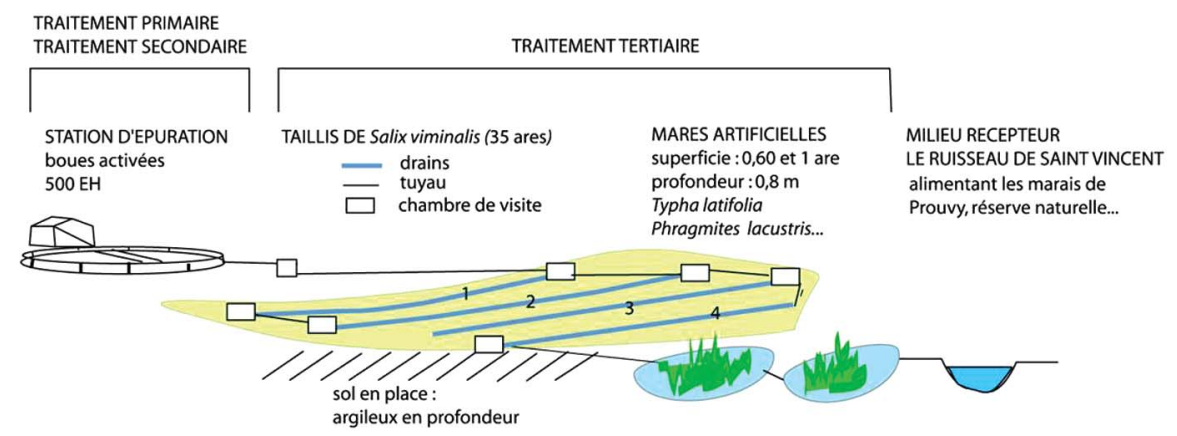

Fig. 3. Schéma de l'installation du taillis de saules à Saint-Vincent.

Fig. 3. Willows coppice plan at Saint-Vincent.

\section{- Dispositifs d'abreuvement du bétail en cours d'eau}

Suite à l'inventaire réalisé en 1994 dans le cadre du premier programme d'actions du contrat de rivière Semois, il a été constaté que la première cause d'érosion des cours d'eau en zone agricole était due au piétinement du bétail. S'il est légitime de conserver l'usage « abreuvement du bétail en cours d'eau », il est aussi opportun de concilier cet usage avec les autres fonctions de la rivière, notamment biologique et hydraulique.

Après une enquête auprès des agriculteurs concernés par les points noirs notés lors de l'inventaire, cinq d'entre eux ont marqué leur accord afin de pouvoir tester dans leur prairie divers types d'accès protégé du bétail au cours d'eau et systèmes d'abreuvoirs. Cinq systèmes différents ont été ainsi installés en 2000-2001 :

- aire d'accès protégée par des billes de chemin de fer ;

- aire d'accès protégée par des rondins de mélèze ;

- abreuvoirs alimentés par l'eau du ruisseau prélevée par pompage (énergie fournie par panneaux photovoltaïques) ;

- abreuvoirs alimentés en gravitaire ;

- pompes actionnées par le bétail de type « buvette ».

\section{- Restauration d'un ancien barrage}

Cinq des sept sites de baignade dans la rivière Semois sont localisés en amont d'un ancien barrage. Il s'agit de barrages en pierres sèches datant de plusieurs siècles. À partir du XIII ${ }^{\mathrm{e}}$ siècle, ces barrages ont été implantés afin d'augmenter le niveau d'eau pour l'alimentation d'un bief de moulin à farine ou pour produire l'énergie des scieries. Ces héritages du passé constituent des éléments forts du patrimoine ancien et paysager appréciés sentimentalement par les riverains et les vacanciers qui souhaitent assurer leur maintien.

Mais quel est le rapport entre ces barrages et les zones de baignade ? Le niveau d'eau du tronçon de rivière en amont subit l'influence de la digue du barrage en permettant une profondeur d'eau suffisante pour la pratique de la baignade. Or l'un des sites de baignade en Semois, la zone de baignade d'Herbeumont en amont du barrage du moulin des Nawés, souffrait d'un manque d'eau suite aux importantes 
brèches de la digue qui empêchent d'atteindre en étiage une profondeur suffisante. À la demande de la Commune d'Herbeumont, des travaux de restauration en vue de retrouver un niveau suffisant pour la baignade ont été envisagés.

\section{RÉSULTATS ET DISCUSSION}

\section{Amélioration de la qualité des eaux de baignade}

Vouloir améliorer la qualité des eaux de baignade passe par une bonne connaissance des sources de contamination. Une étude réalisée en 2000 et 2001, pour compte de la Région wallonne (Rosillon et al., 2002), a permis de mettre en évidence les origines des sources de contamination bactérienne des zones de baignade en Wallonie (voir tab. I).

Lors de cette étude, nous avons donc examiné la situation des 33 zones récréatives existantes en Wallonie. Près de la moitié des principales causes de dégradation trouvaient leur origine dans l'impact des rejets d'agglomérations en épuration collective (26\%) et d'installations touristiques en bordure de cours d'eau (23\%). Signalons qu'à cette époque, peu de stations d'épuration sont complétées par un dispositif de désinfection. Par ailleurs, l'apport de contaminants fécaux par l'agriculture intervenait dans $9 \%$ des causes de dégradation de la qualité bactériologique des zones de baignade.

Les deux actions, présentées ci-après, répondent à deux des problématiques évoquées ci-dessus, à savoir l'épuration des eaux domestiques et la diminution de l'impact du bétail.

\section{Résultats obtenus à partir des trois dispositifs mis en place}

\section{- Épuration des eaux usées domestiques par taillis de saules : un système d'épuration innovant pour l'abattement des indicateurs de contamination fécale}

Après les premières années de fonctionnement du taillis de saules, les abattements des formes de l'azote sont satisfaisants, les rendements sont de l'ordre de $70 \%$. Par contre, les abattements en phosphore, avec un rendement de $50 \%$, restent encore en deçà des espérances de départ ; les temps de séjour étant relativement courts. Concernant les contaminants fécaux, les concentrations moyennes observées en sortie du système sont de l'ordre de 300 à 450 E. coli par $100 \mathrm{~mL}$ et de 30 à $50 \mathrm{E}$. fécaux par $100 \mathrm{~mL}$. Alors qu'à l'entrée (sortie de la step par boues activées), les normes ne sont pas respectées, notamment la norme impérative de la directive 76/160/CEE qui fixe le seuil à 2000 coliformes fécaux par $100 \mathrm{~mL}$, en sortie la qualité correspond à une excellente qualité selon la directive 2006/7/CE (voir tab. II et III).

Dès l'instant où le terrain est disponible, et particulièrement en milieu rural, ce dispositif extensif pour petite collectivité peut garantir une excellente qualité bactériologique des eaux du cours d'eau récepteur propice à la pratique de la baignade.

\section{- Bétail et qualité bactériologique des zones de baignade}

Dans le cadre de l'évaluation des dispositifs décrits précédemment et mis en place en vue de réduire l'impact du bétail sur les cours d'eau, une étude comparative 
Tableau I. Origine des sources de contamination des 33 zones de baignade en Région wallonne.

Table I. Origin of pollution sources of 33 bathing sites in the Wallon Region.

\begin{tabular}{|lc|}
\hline Origine des contaminations & Pourcentage de cas observés \\
\hline Impact par temps de pluie & $8 \%$ \\
Stations d'épuration & $7 \%$ \\
Entités en épuration collective & $26 \%$ \\
Installations touristiques & $23 \%$ \\
Entités en épuration individuelle & $17 \%$ \\
Apports d'origine agricole & $9 \%$ \\
Autres & $10 \%$ \\
\hline
\end{tabular}

Tableau II. Performance d'un taillis de saules sur la réduction des contaminants fécaux, campagnes 2003-2004.

Table II. Results of a willows coppice on the reduction of the feacal bacteria (2003-2004).

\begin{tabular}{|c|c|c|c|c|c|}
\hline \multirow[b]{2}{*}{$\begin{array}{l}\text { Contaminants } \\
\text { fécaux }\end{array}$} & \multicolumn{2}{|c|}{ Entrée du taillis } & $\begin{array}{c}\text { Abattement } \\
\text { entrée/sortie } \\
\text { du taillis }\end{array}$ & $\begin{array}{c}\text { Abattement } \\
\text { entrée/sortie } \\
\text { des mares }\end{array}$ & $\begin{array}{l}\text { Abattement } \\
\text { total }\end{array}$ \\
\hline & $\begin{array}{c}\text { Moyenne } \\
\text { CFU/ } \\
100 \mathrm{~mL}\end{array}$ & $\begin{array}{c}\text { Min-Max } \\
\text { CFU/100 mL }\end{array}$ & $(\%)$ & $(\%)$ & $(\%)$ \\
\hline $\begin{array}{l}\text { Escherichia } \\
\text { coli }\end{array}$ & 60900 & $(2100-106700)$ & $74-98$ & $67-93$ & $98,9-99,3$ \\
\hline $\begin{array}{l}\text { Entérocoques } \\
\text { fécaux }\end{array}$ & 10500 & $(4400-18600)$ & $68-85$ & $98,4-98,9$ & $99,5-99,8$ \\
\hline
\end{tabular}

Tableau III. Performance d'un taillis de saules sur la réduction des contaminants fécaux, campagne 2008.

Table III. Results of a willows coppice on the reduction of the feacal bacteria (2008).

\begin{tabular}{|lccc|}
\hline & \multicolumn{2}{c|}{$\begin{array}{c}\text { Abattement } \\
\text { entrée/sortie } \\
\text { du dispositif } \\
\text { taillis + mares }\end{array}$} \\
\cline { 2 - 4 } Contaminants fécaux & Entrée du taillis & $\begin{array}{c}\text { Min-Max } \\
\text { Moyenne }\end{array}$ & $\begin{array}{c}\text { CFU/100 mL } \\
\text { Escherichia coli }\end{array}$ \\
Entérocoques fécaux & 57440 & $(16800-119000)$ & 99,5 \\
\hline
\end{tabular}




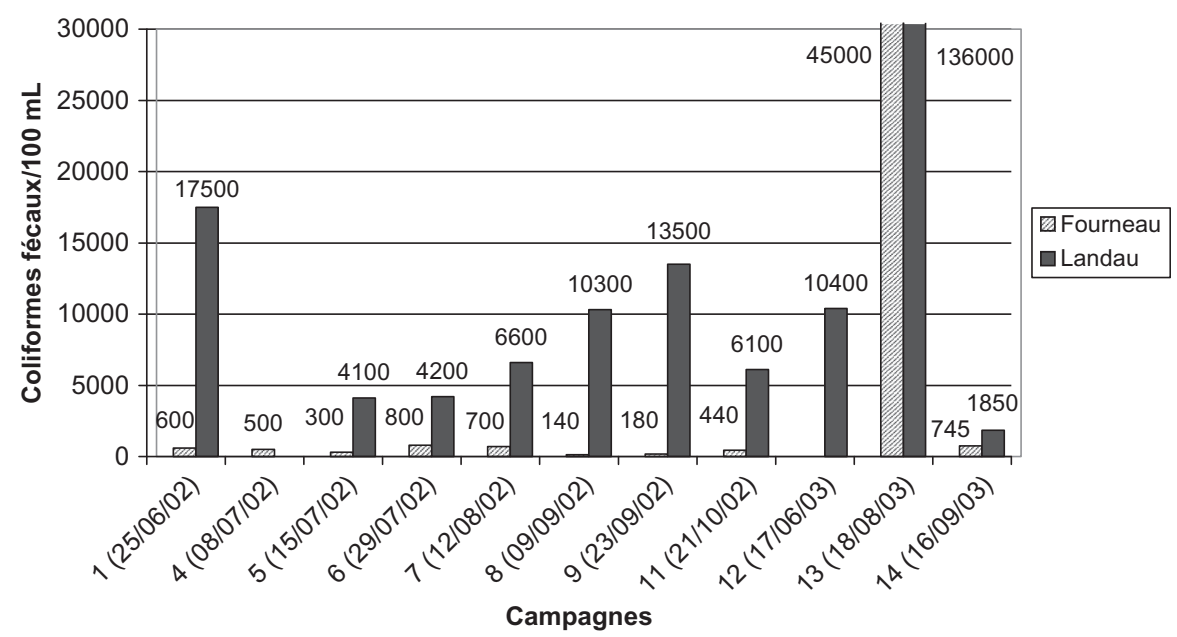

Fig. 4. Évolution et comparaison de la concentration en coliformes fécaux dans deux ruisseaux soumis à l'abreuvement du bétail.

Fourneau : Ruisseau avec abreuvement du bétail contrôlé.

Landau : Ruisseau avec accès libre du bétail.

Fig. 4. Evolution and comparison of the faecal coliforms concentration in two watercourses with cattle watering.

Fourneau: River with cattle watering controlled.

Landau: River with free access cattle.

de la qualité de deux affluents voisins de la Rulles, l'un protégé (le Fourneau) et l'autre où le bétail continue à divaguer (le Landau) a montré l'intérêt de ce type d'installation en faveur de la qualité physico-chimique et biologique de la rivière (Rosillon et al., 2005a). Les résultats d'analyse confirment la bonne qualité du ruisseau protégé par une clôture où l'abreuvement du bétail est contrôlé (le Fourneau), par rapport au ruisseau où l'accès du bétail est libre (le Landau). Pour ce dernier, les valeurs en ammonium, nitrites et phosphates sont deux à trois fois plus élevées. L'indice biologique global normalisé (IBGN) passe de 9 pour le Landau à 15 pour le Fourneau. Signalons également que les capacités d'accueil pour le frai de la truite sont deux fois plus importantes dans le Fourneau que dans le Landau.

Mais au-delà de ces bons résultats de qualité physico-chimique et écologique, un abreuvement contrôlé en cours d'eau permet, en empêchant la défécation directe dans la rivière, de réduire fortement les apports en contaminants fécaux lorsque l'on sait qu'une bouse de vache peut contenir des millions de bactéries fécales.

Indépendamment d'un épisode pluvieux qui a perturbé fortement la qualité bactériologique des deux ruisseaux, les valeurs observées dans le cours d'eau protégé (de 140 à $800 \mathrm{E}$. coli/100 $\mathrm{mL}$ ) correspondent à une excellente à bonne qualité selon la directive baignade de 2006. En revanche, le cours d'eau non protégé présente des concentration de 1850 à $17500 \mathrm{E}$. coli/100 mL qui disqualifie le cours d'eau en tant que zone de baignade (voir fig. 4).

Tout en maintenant l'abreuvement du bétail en cours d'eau, la mise en place de dispositifs de protection et d'aires d'accès protégés permettent de concilier dans 


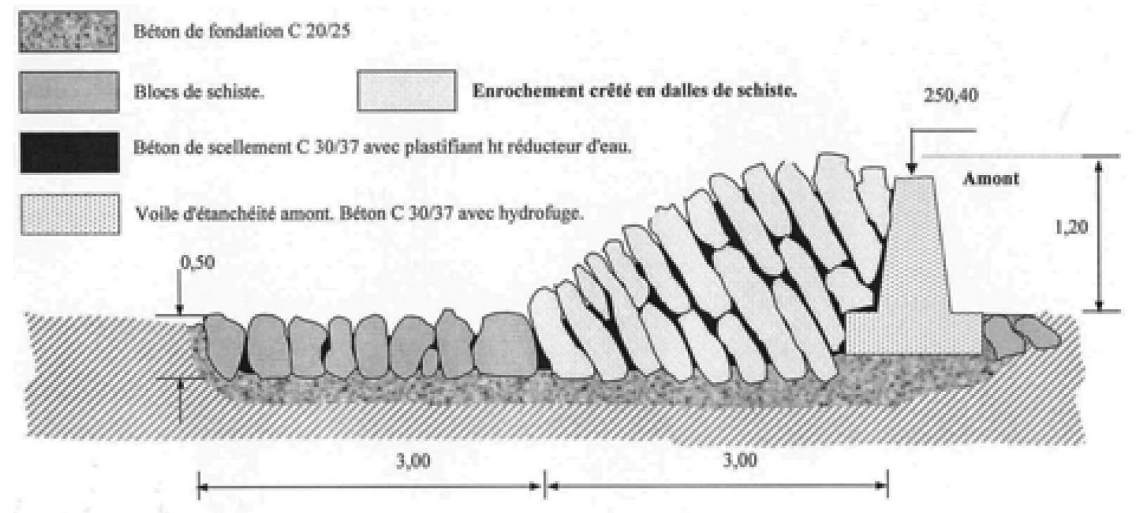

\section{Radier aval Déversoir Voile d'étanchéité}

Fig. 5. Restauration du barrage des Nawés à Herbeumont, profil en travers (Ministère de la Région wallonne, Daxhelet C., 2006).

Fig. 5. Restoration of a dam at Herbeumont, across profile (Ministry of the Walloon Region, Daxhelet C., 2006).

un même cours d'eau, abreuvement du bétail et pratique de la baignade tout en assurant la protection du milieu en vue d'atteindre le bon état écologique exigé par la Directive Cadre Eau (DCE).

\section{- Aspects quantitatifs : la restauration d'un ancien barrage, quand «baignade» et «patrimoine» se conjuguent}

Les travaux de restauration du barrage des Nawés (Commune d'Herbeumont) ont été précisés lors de différentes concertations entre acteurs de la rivière (secteur touristique, pêcheurs, naturalistes, services de la Région wallonne, gestionnaire du cours d'eau. ..). Les techniques mises en œuvre ont permis de prendre en compte les préoccupations de chacun des acteurs en intégrant les différents usages et fonctions du cours d'eau à cet endroit (voir fig. 5).

Les travaux ont porté sur la construction d'une nouvelle digue en tenant compte des aspects suivants :

- en priorité l'usage baignade par la mise en place d'un voile étanche en béton afin de garantir le niveau d'eau même par faible débit en période estivale ;

- la fonction patrimoniale et paysagère : en masquant cet élément artificiel par une structure en pierres mimant la technique ancienne dite de perrés crêtés en pierres sèches ;

- la fonction piscicole, par l'installation d'une échelle à poissons ;

- la fonction récréative, par le placement d'une passe à kayak ;

- la fonction hydraulique, par la restauration du bief d'alimentation du moulin.

De plus, le site étant localisé en zone protégée Natura 2000, le respect des écosystèmes inféodés à ce barrage (îles à grande biodiversité liées à ce barrage) s'imposait. 


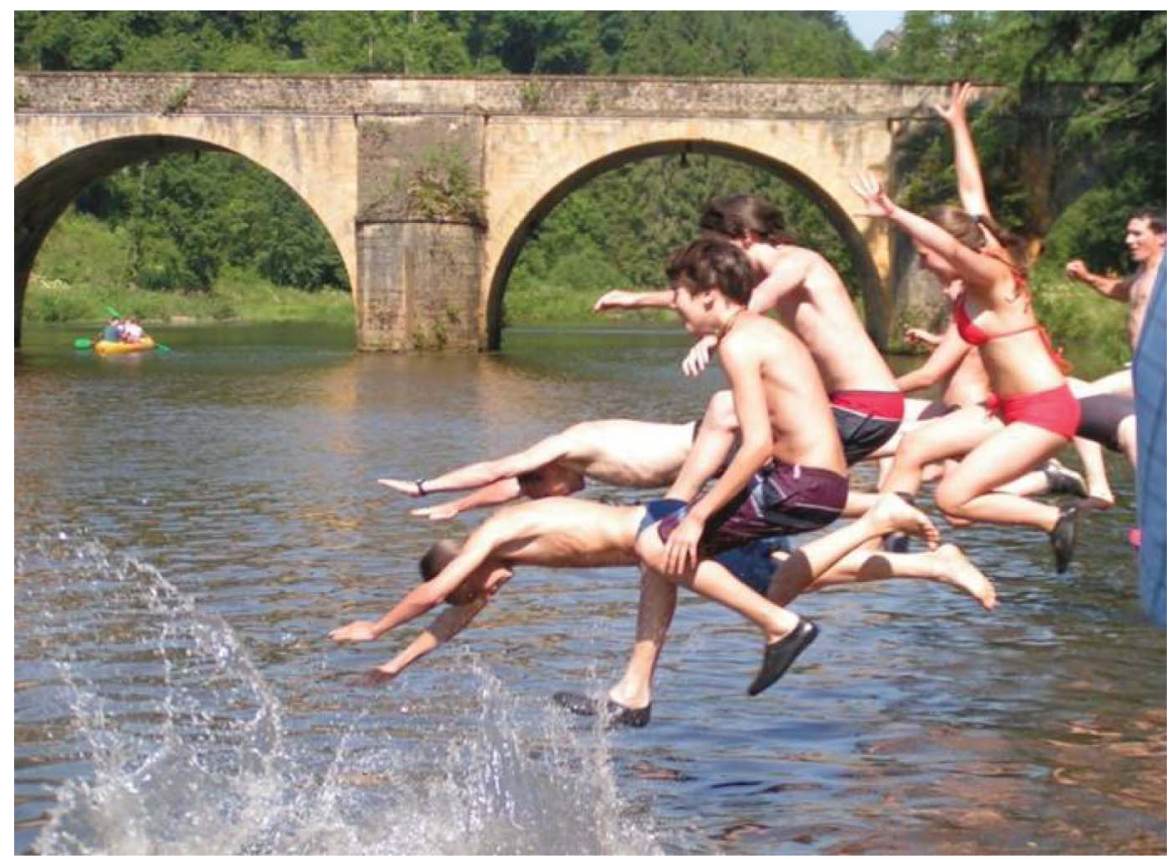

Fig. 6. Big Jump en Semois (juillet 2005).

Fig. 6. Big Jump in the Semois river (July 2005).

Désormais, le niveau d'eau retrouvé (plus d'un mètre) permet de pratiquer la baignade dans ce site officiellement reconnu par la Région wallonne.

Cet exemple d'action illustre aussi très bien le caractère multifonctionnel de la rivière et la prise en compte de l'usage baignade qui conduisent les gestionnaires des cours d'eau à concilier diverses préoccupations en vue d'une gestion intégrée.

\section{Vers une reconquête citoyenne de la rivière}

La baignade en Semois, dans l'esprit du contrat de rivière, est une opportunité locale très appréciée et conduit à une réappropriation sociale de la rivière. Ce sentiment d'appartenance à une rivière est renforcé lors de l'organisation annuelle des journées européennes de la baignade, qui se déroulent chaque année en Région wallonne. Il s'agit de l'opération « Big Jump » au cours de laquelle, le même jour, les citoyens européens sont invités au grand plongeon dans les rivières (voir fig. 6). Cette manifestation festive est l'occasion pour les citoyens de se réapproprier leur environnement et de montrer leur volonté de retrouver des rivières propres et vivantes ${ }^{3}$. 


\section{CONCLUSION}

Milieu complexe et multifonctionnel, la rivière offre un espace de rencontre à divers usagers dont les adeptes de la baignade. Mais le plaisir de pratiquer ce loisir en milieu naturel en un lieu bucolique au milieu des poissons et des nénuphars peut être contrarié par des éléments perturbateurs tels qu'un rejet d'eaux usées domestiques, la présence de bétail s'abreuvant au cours d'eau en amont de la zone de baignade, ou encore par un trop faible niveau d'eau transformant la baignade en pataugeoire pour bain de pieds.

À travers la mise en œuvre d'un programme d'actions inscrites dans le contrat de rivière Semois, nous avons pu montrer que par une gestion intégrée et concertée, tous ces aspects reliés entre eux pouvaient être pris en compte.

De par les exigences de qualité d'eau qu'elle réclame, la baignade en rivière peut servir d'élément fédérateur pour catalyser les efforts pour sa restauration; des efforts en matière d'épuration ou en vue de réduire l'impact de l'abreuvement du bétail en cours d'eau, mais aussi à travers des aménagements hydrauliques au service de la pratique de la baignade.

Ces efforts ne servent pas seulement l'usage baignade, tout l'écosystème est gagnant, et au final ces actions devraient permettre de tendre vers le « bon état » de qualité des eaux exigé par la DCE à l'échéance 2015.

Les trois cas présentés, qui ont donc été initiés dans le cadre d'un contrat de rivière, permettent de démontrer l'intérêt et la possibilité de concilier divers usages de l'eau et de répondre aux préoccupations des usagers, notamment des adeptes de la baignade en rivière. Les deux premières actions ont conduit à une amélioration de la qualité bactériologique des eaux de baignade à travers un dispositif original de désinfection d'eaux usées domestiques par taillis de saules et la mise en place d'abreuvoirs pour bétail afin de limiter la contamination fécale en provenance des bovins. La troisième réalisation a permis le maintien d'un niveau d'eau suffisant pour la pratique de la baignade en amont d'un ancien barrage qui a fait l'objet d'une restauration.

Rappelons enfin qu'à travers des actions de sensibilisation comme l'opération « Big Jump », cette pratique populaire qu'est la baignade en milieu naturel peut constituer un excellent support de sensibilisation du grand public à la bonne qualité des eaux de surface, tout en apportant un soutien citoyen à la mise en œuvre de la directive cadre eau.

\section{RÉFÉRENCES}

Gouvernement wallon, 2008. Arrêté du Gouvernement wallon modifiant le livre II du code de l'environnement contenant le code de l'eau, relatif aux contrats de rivière adopté le 13 novembre 2008.

GREEN asbl et Inter-Environnement Wallonie, 2009. Site web du Big Jump en Belgique, http://www.bigjump.be.

Journal officiel de l'Union européenne, 2006. Directive 2006/7/CE du parlement européen et du conseil du 15 février 2006 concernant la gestion de la qualité des eaux de baignade et abrogeant la directive 46/160/CEE. Journal officiel de l'Union européenne du 4.3.2006, L64/37-L64/51.

Lutgen B., 2009. Nouvelles mesures pour améliorer la qualité des eaux de baignade. Cabinet du Ministre, http://lutgen.wallonie.be/spip/spip.php?article745, consulté le 16/09/2009. 
Ministère de l'écologie, de l'énergie, du développement durable et de la mer, Office International de l'Eau, Agences de l'eau, 2009. GEST'EAU, le portail national de l'eau : le site des outils de gestion intégrée de l'eau, les contrats de rivière. http://www.gesteau.eaufrance.fr/.

Ministère de la Région wallonne, 2006. Projet de restauration du barrage des Nawés à Herbeumont, cahier des charges, Direction des Cours d'eau non navigables, Service extérieur de Marche, Daxhelet C.

Rosillon F. et Vander Borght P., 2005b. Les contrats de rivière en Wallonie (Belgique) : retour d'expériences. Revue Européenne de droit de l'Environnement, 4-2004, 414-429.

Rosillon F., Culot M., Bosser C., Vaerewyck J.F. et Vander Borght P., 2002. Contribution à l'étude de la qualité bactériologique des eaux de surface à vocation touristique en Wallonie (Belgique). Cahiers de l'Association Scientifique Européenne pour l'Eau et la Santé, 7, 1, 3-13.

Rosillon F., Vander Borght P., Ledermann C., Van Doren B., 2004. Willows Short Rotation Coppice (WSRC) for waste water purification and protection of receiver watercourses. Conférences Internationales ASTEE-IWA, Avignon 4 p.

Rosillon F., Vander Borght P., Vanhees V., Causse E. et Giot B., 2005a. Impact de l'abreuvement du bétail sur la qualité écologique des cours d'eau : étude de cas en Région wallonne de Belgique. Cahiers de l'Association Scientifique Européenne pour l'Eau et la Santé, 10, 1, 59-70.

Rosillon F., Lobet J., Tassin M. et Vander Borght P., 2009. La Semois et son bassin, histoire d'un contrat de rivière. In: Au fil de la Semois, Cahier de l'Académie luxembourgeoise, 23/2009, 29-59.

Service Public de Wallonie, 2008. Tableau de bord de l'environnement wallon, 2008. Direction générale opérationnelle Agriculture, ressources naturelles et Environnement, Cellule État de l'Environnement wallon, Éd. SPW, 199 p.

Service Public de Wallonie, 2009. Directive-cadre sur l'eau, Programme de mesures, zones protégées, zones de baignade. http://environnement.wallonie.be/directive_eau/pg/7/75//755. asp, consulté le 16/09/2009.

Simonet F., 2001. Le nouveau système d'évaluation de la qualité de l'eau des rivières : le SEQ-EAU. Revue de l'Agence de l'eau Adour-Garonne, hiver 2001, 81, 7-9.

Site web des contrats de rivière en Région wallonne de Belgique, 2009. http://environnement. wallonie.be/contra_riviere/.

Site web du contrat de rivière Semois, 2009. http://www.semois-semoy.org. 